\title{
On multicomponent effects in stellar winds of stars at extremely low metallicity
}

\author{
J. Krtička ${ }^{1,2,3}$, S. P. Owocki ${ }^{1,4}$, J. Kubát ${ }^{3}$, R. K. Galloway ${ }^{1}$, and J. C. Brown ${ }^{1}$ \\ 1 Department of Physics and Astronomy, University of Glasgow, Glasgow G12 8QQ, UK \\ 2 Ústav teoretické fyziky a astrofyziky PřF MU, 61137 Brno, Czech Republic \\ 3 Astronomický ústav, Akademie věd České republiky, 25165 Ondřejov, Czech Republic \\ ${ }^{4}$ Bartol Research Institute, University of Delaware, Newark, DE 19716, USA
}

Received 29 November 2002 / Accepted 20 February 2003

\begin{abstract}
We calculate multicomponent line-driven wind models of stars at extremely low metallicity suitable for massive first generation stars. For most of the models we find that the multicomponent wind nature is not important for either wind dynamics or for wind temperature stratification. However, for stars with the lowest metallicities we find that multicomponent effects influence the wind structure. These effects range from pure heating to possible fallback of the nonabsorbing wind component. We present a simple formula for the calculation of metallicity for which the multicomponent effects become important. We show that the importance of the multicomponent nature of winds of low metallicity stars is characterised not only by the low density of driving ions, but also by lower mass-loss rate.
\end{abstract}

Key words. stars: mass-loss - stars: early-type - stars:abundances - stars: winds, outflows

\section{Introduction}

First generation stars are a textbook example of the importance of initial metallicity for stellar structure and evolution. During the gravitational collapse of extremely-low metallicity clouds the formation of massive stars was possibly much more favored than in the present time (cf. Bromm et al. 2002; Nakamura \& Umemura 2002) and the evolution of such massive stars was strikingly different from standard stellar evolution (Siess et al. 2002). Similarly, due to their enormous luminosity and minute abundance of elements heavier than helium, their line-driven winds substantially differed from those of present day stars.

The first models of line-driven stellar winds suitable for very massive first generation stars were calculated by Kudritzki (2002). He concluded that these stars must be near to the Eddington limit in order to possess line-driven winds. However, as was discussed by Kudritzki, in the case of stars at such extremely low metallicity other effects may become important. It is well known that radiative driven stellar winds have a multicomponent nature (cf. Castor et al. 1976), the reason for which is relatively straightforward. The radiative force accelerating a stellar wind is distributed unevenly over individual ions. Whereas minor absorbing elements, such as $\mathrm{C}, \mathrm{N}, \mathrm{O}$ or Fe, obtain momentum from the stellar radiation field, hydrogen and helium are only marginally accelerated by the radiation. However, these nonabsorbing components are accelerated

Send offprint requests to: J. Krtička,

e-mail: jiri@astro.gla.ac.uk by friction with the absorbing components. This momentum transfer between low-density absorbing and high-density nonabsorbing components is specially important for low-density stellar winds (Springmann \& Pauldrach 1992; Krtička \& Kubát 2001; hereafter KKII). These stellar winds can be heated by the friction between components and, for extremely low wind density, even hydrogen fallback may occur. Low density stellar winds may also be subject to so called runaway instability (see Owocki \& Puls 2002; Krtička \& Kubát 2002). This instability occurs when the velocity difference between absorbing and nonabsorbing components is comparable to the averaged sound speed (Krtička \& Kubát 2001, Eq. (45)). Finally, low-density stellar winds are heated by the so-called GayleyOwocki heating (Gayley \& Owocki 1994, hereafter GO). This heating/cooling is caused by the dependence of the radiative force on the velocity via the Doppler effect.

Because these multicomponent effects usually occur mainly in the outer part of the wind downstream from the critical point they do not affect the mass-loss rate but they influence the wind temperature and outflow velocity. In this paper we discuss these multicomponent effects for stars at extremely low metallicity.

\section{Domain of importance of multicomponent effects}

In order to assess the importance of multicomponent effects we use a simple formula for the velocity difference 
between absorbing and nonabsorbing components. Similarly to Springmann \& Pauldrach (1992) and Owocki \& Puls (2002) we start from the equation of motion of nonabsorbing component for a two-component wind. Neglecting the gravitational acceleration, electrical force and gas pressure term, this equation takes the form (see KKII)

$v_{r \mathrm{p}} \frac{\mathrm{d} v_{r \mathrm{p}}}{\mathrm{d} r}=\frac{\rho_{\mathrm{i}}}{m_{\mathrm{p}} m_{\mathrm{i}}} \frac{4 \pi q_{\mathrm{p}}^{2} q_{\mathrm{i}}^{2}}{k T} \ln \Lambda G\left(x_{\mathrm{pi}}\right)$,

where $v_{r \mathrm{p}}$ is the velocity of the nonabsorbing component. For the calculation of frictional acceleration on the right-hand side of Eq. (1) we assume that the temperatures of all components are nearly equal $\left(T_{\mathrm{p}} \approx T_{\mathrm{i}} \approx T\right)$, and $\rho_{\mathrm{i}}$ is the mass density of the absorbing component, $m_{\mathrm{p}}, m_{\mathrm{i}}, q_{\mathrm{p}}$ and $q_{\mathrm{i}}$ are the particle masses and charges of nonabsorbing and absorbing components, and $\ln \Lambda$ is the Coulomb logarithm. The Chandrasekhar function $G\left(x_{\mathrm{pi}}\right)$ is defined in terms of the error function $\operatorname{erf}(x)$ (cf. Burgers 1969)

$$
\begin{aligned}
& G\left(x_{\mathrm{pi}}\right)=\frac{1}{2 x_{\mathrm{pi}}^{2}}\left(\operatorname{erf}\left(x_{\mathrm{pi}}\right)-\frac{2 x_{\mathrm{pi}}}{\sqrt{\pi}} \exp \left(-x_{\mathrm{pi}}^{2}\right)\right), \\
& x_{\mathrm{pi}} \approx \frac{v_{r \mathrm{i}}-v_{r \mathrm{p}}}{\sqrt{\frac{2 k T}{m_{\mathrm{p}}}}} .
\end{aligned}
$$

The Chandrasekhar function can be approximated for $x_{\mathrm{pi}}<$ 1 by

$$
G\left(x_{\mathrm{pi}}\right) \approx \frac{2 x_{\mathrm{pi}}}{3 \sqrt{\pi}} .
$$

Using a $\beta$-velocity law $v_{r \mathrm{p}}=v_{\infty}\left(1-\frac{R_{*}}{r}\right)$ (with $\beta=1$, which is near to the mean observed value, cf. Puls et al. 1996) for the non-absorbing component we can approximate $\frac{\mathrm{d} v_{\mathrm{pp}}}{\mathrm{d} r} \approx v_{\infty} \frac{R_{*}}{r^{2}}$. The continuity equation can be used to calculate the density of absorbing ions

$\rho_{\mathrm{i}} \approx\left(\frac{Z}{Z_{\odot}}\right) \mathfrak{Y}_{\mathrm{i}} \rho_{\mathrm{p}} \approx \frac{1}{4 \pi r^{2} v_{r \mathrm{p}}}\left(\frac{Z}{Z_{\odot}}\right) \mathfrak{Y}_{\mathrm{i}} \dot{\mathfrak{M}}$,

where $v_{\infty}$ and $R_{*}$ are the terminal velocity and stellar radius, $\mathfrak{Y}_{\mathrm{i}}$ is the mass density ratio of absorbing and nonabsorbing ions in the solar photosphere $\left(\mathfrak{Y}_{\mathrm{i}}=0.0127\right.$, this value corresponds to the solar ratio of sum of densities of $\mathrm{C}, \mathrm{N}, \mathrm{O}, \mathrm{Fe}$ to the density of bulk plasma), $Z / Z_{\odot}$ is the metallicity (number density of absorbing ions relative to hydrogen) in the stellar atmosphere relative to the solar value, and $\dot{\mathfrak{M}}$ is mass loss rate. Solving the momentum Eq. (1) for the velocity difference we obtain

$$
\frac{v_{r \mathrm{i}}-v_{r \mathrm{p}}}{\sqrt{\frac{2 k T}{m_{\mathrm{p}}}}} \approx v_{r \mathrm{p}}^{2} \frac{v_{\infty} R_{*}}{\left(Z / Z_{\odot}\right) \dot{\mathfrak{M}}} \frac{3 \sqrt{\pi} m_{\mathrm{p}} m_{\mathrm{i}} k T}{2 \mathfrak{Y}_{\mathrm{i}} q_{\mathrm{p}}^{2} q_{\mathrm{i}}^{2} \ln \Lambda} .
$$

Apparently, as the wind is accelerated the velocity difference increases. Thus, multicomponent effects are important mainly in the outer part of the wind. If the wind temperature is constant then the maximal velocity difference is attained for maximal radial velocity, i.e. for $v_{r \mathrm{p}}=v_{\infty}$. This enables us to calculate the maximal velocity difference between wind components for each model star.

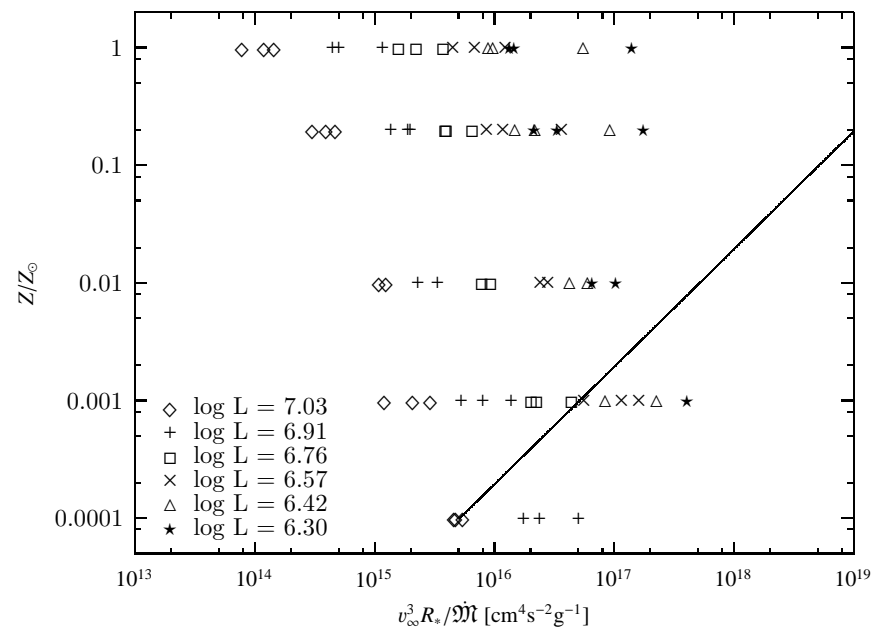

Fig. 1. The domain of importance of multicomponent effects. Each model star is represented by a point in this plot. The straight line (calculated using Eq. (6), where we inserted $v_{r \mathrm{p}}=v_{\infty}$ ) divides regions where multicomponent effects are negligible from where they are important. Winds of stars above this line have a low velocity difference between wind components whereas for stars below the line multicomponent effects are important. Different symbols denote different stellar luminosity (given in solar units).

The multicomponent effects are found to be important when the nondimensional velocity difference is typically

$\frac{v_{r \mathrm{i}}-v_{r \mathrm{p}}}{\sqrt{\frac{2 k T}{m_{\mathrm{p}}}}} \gtrsim 0.1$

(KKII). In this case frictional heating influences the wind temperature. Note that there is some kind of feedback because the higher temperature enhances the multicomponent effects. In the plot of metallicity versus the quantity $v_{\infty}^{3} R_{*} / \dot{\mathfrak{M}}$ (see Fig. 1) we shall use a straight line to divide regions where multicomponent effects are important/unimportant. Stars above this line have a low relative velocity difference between wind components (see Eq. (6)) whereas stars below this line have higher relative velocity difference and thus multicomponent effects are important for these stars. The dividing line (for which $\left.x_{\mathrm{pi}}=0.1\right)$ was plotted with assumed average wind temperature $T=25000 \mathrm{~K}$, ionic charges $q_{\mathrm{p}}=0.85 e, q_{\mathrm{i}}=4.0 e$ (where $e$ is the electronic charge) and the mean mass of absorbing ions corresponding to carbon.

We added each star for which Kudritzki (2002) calculated a wind model into Fig. 1. We used the same wind parameters (i.e. wind temperature, ionic charges and mass of absorbing ions) as in the previous paragraph for the plot of dividing line. There are several stars for which the multicomponent effects are important. For stars with highest luminosity only the models with lowest metallicity may suffer from the multicomponent effect. The lower the luminosity, the lower the mass loss rate and the higher is the metallicity for which multicomponent effect becomes important. Moreover, for stars with given basic parameters (i.e. mass, radii and effective temperature) we are able to find a value of metallicity for which the multicomponent effects influence the wind structure. This conclusion is especially important for low metallicity stars. 
For normal solar-type ion abundance with $\left(Z / Z_{\odot}\right) \approx 1$ Eq. (6) indicates that ion runaway should occur only for very low mass loss rates, i.e. $\dot{\mathfrak{M}} \sim 10^{-11} M_{\odot} \mathrm{yr}^{-1}$, and so is relevant only for relatively low luminosity stars. But for stars with very low metallicity, ion runaway could become important for quite luminous stars. Consider, for example, the canonical CAK mass loss scaling (see Pauldrach et al. 1986)

$\dot{\mathfrak{M}} \approx \frac{L}{c^{2}} \frac{\alpha}{(1-\alpha)(1+\alpha)^{1 / \alpha}}\left[\frac{\bar{Q} \Gamma}{1-\Gamma}\right]^{-1+1 / \alpha}$,

where $\Gamma$ is the Eddington parameter, and $\bar{Q} \approx 2000 \mathrm{Z} / \mathrm{Z}_{\odot}$ (Gayley 1995). Because $\alpha<1$ (Abbott 1982; Puls et al. 2000) this generally means that lower metallicity enables larger multicomponent effects not only due to the lower abundance of the driving ions but also due to the lower mass-loss rate (see Eq. (6)). The mass-loss rate formula (7) can be rewritten using the scaled quantities $\dot{M}_{-11} \equiv \dot{\mathfrak{M}} /\left(10^{-11} \mathfrak{M}_{\odot} \mathrm{yr}^{-1}\right)$, $M_{2} \equiv \mathfrak{M} / 10^{2} \mathfrak{M}_{\odot}, L_{6} \equiv L / 10^{6} L_{\odot}$, and $\Gamma \approx 0.26 L_{6} / M_{2}$. For the canonical case of $\alpha=2 / 3$ and $\Gamma=0.5$, this yields

$\dot{M}_{-11} \approx 2.1 \times 10^{5} \frac{L_{6}^{3 / 2}}{M_{2}^{1 / 2}}\left(\frac{Z}{Z_{\odot}}\right)^{1 / 2}$.

Similarly, Eq. (6) implies that the maximal velocity difference scales as

$x_{\mathrm{pi}} \approx 1.2 \frac{v_{8}^{3} R_{12} T_{4}}{\dot{M}_{-11}}\left(\frac{Z}{Z_{\odot}}\right)^{-1}$

where $v_{8} \equiv v_{\infty} /\left(10^{8} \mathrm{~cm} \mathrm{~s}^{-1}\right), R_{12}=R_{*} / 10^{12} \mathrm{~cm}$, and $T_{4} \equiv$ $T / 10^{4} \mathrm{~K}$. Recalling that multicomponent effects are important for $x_{\mathrm{pi}} \gtrsim 0.1$, then inserting this value into (9) we can obtain the minimal metallicity $Z_{(1)}$ for which one-component models are applicable. By application of (8) in (9) we find that

$\left(\frac{Z_{(1)}}{Z_{\odot}}\right) \approx 1.5 \times 10^{-3} \frac{v_{8}^{2} R_{12}^{2 / 3} T_{4}^{2 / 3} M_{2}^{1 / 3}}{L_{6}}$.

The latter equation can be rewritten in terms of basic stellar parameters using a canonical estimate of the wind terminal velocity $v_{\infty}^{2}=\alpha /(1-\alpha) v_{\text {esc }}^{2}$ (see Castor et al. 1975, hereafter CAK), where $v_{\text {esc }}$ is the escape velocity. Thus, the lowest metallicity for which one-component wind models can be applied scales as

$\left(\frac{Z_{(1)}}{Z_{\odot}}\right) \approx 4 \times 10^{-3} \frac{T_{4}^{2 / 3} M_{2}^{4 / 3}}{R_{12}^{1 / 3} L_{6}}$.

In any case, the general result from Eq. (11) is that multicomponent effects are likely to play a role only in very low metallicity massive stars.

\section{Model description}

To test this scaling analysis we calculated multicomponent models of stars initially modelled by Kudritzki (2002) and looked for multicomponent effects. In order to obtain correct models for massive stars at extremely low metallicity and to improve the model convergence we used slightly different models from KKII. These changes are not important for the final results since these models retain basics characteristics of the KKII models.

We assume that a stellar wind consists of three components, namely absorbing driving ions, passive nonabsorbing ions (hydrogen and helium, which mostly contribute to the bulk wind density) and electrons. For absorbing and nonabsorbing ions we solve the continuity equations, momentum equations, and energy equations. The most important terms in the momentum equations are the radiative and frictional acceleration, while the most important terms in the energy equation are the heat exchange between wind components and the GO heating. Radiative force is calculated using the Sobolev approximation (CAK) with finite disk correction factor (Friend \& Abbott 1986; Pauldrach et al. 1986). Contrary to KKII, for the electrons we solve the energy equation only. The radiative heating/cooling term in this equation is calculated using thermal balance of electrons (Kubát et al. 1999). Stellar fluxes at the lower boundary of the wind were determined using models calculated by a code of Kubát (2002). Electron density and velocity are calculated from the conditions of electrical neutrality and zero current. The charge of the nonabsorbing component is calculated using ionization balance of hydrogen and helium. We included helium to obtain the correct electron density. This proved to be crucially important in getting correct results for stars near the Eddington limit. Finally, we included new lineforce multipliers introduced by Kudritzki (2002) both into the solved equations (with inclusion of the critical point condition) and into the linearization matrix (see Krtička 2002).

The output of these models is the hydrodynamic structure of a three-component radiatively driven stellar wind, i.e. density, velocity, temperature and charge of all components. For a more detailed description of these models see KKII.

Originally, for the calculation of the radiative force CAK introduced a parameter related to the thermal velocity, calculated at the stellar effective temperature. However, the radiative force itself does not depend on the thermal velocity. KKII assumed an artificial dependency of this thermal velocity parameter on the local temperature instead of on the stellar effective temperature. To come closer to models calculated by Kudritzki (2002) and to obtain a correct value of radiative force we assumed that the thermal velocity parameter used for the calculation of radiative force is constant.

The temperature dependence of the radiative force has, however, an important impact on the calculated models. If the radiative force does not depend significantly on the wind temperature (as was consistently assumed in this paper) then the velocity difference between wind components can reach the sound speed and runaway instability occurs. On the other hand, if the radiative force is decreasing with increasing temperature (as was assumed by KKII) then only heating occurs, and is accompanied by the lowering of the terminal velocity, or by the backfall of hydrogen for stars with extremely low-density winds.

\section{Calculated models}

Generally, calculated models where multicomponent effects are important can be divided into three groups according to the 

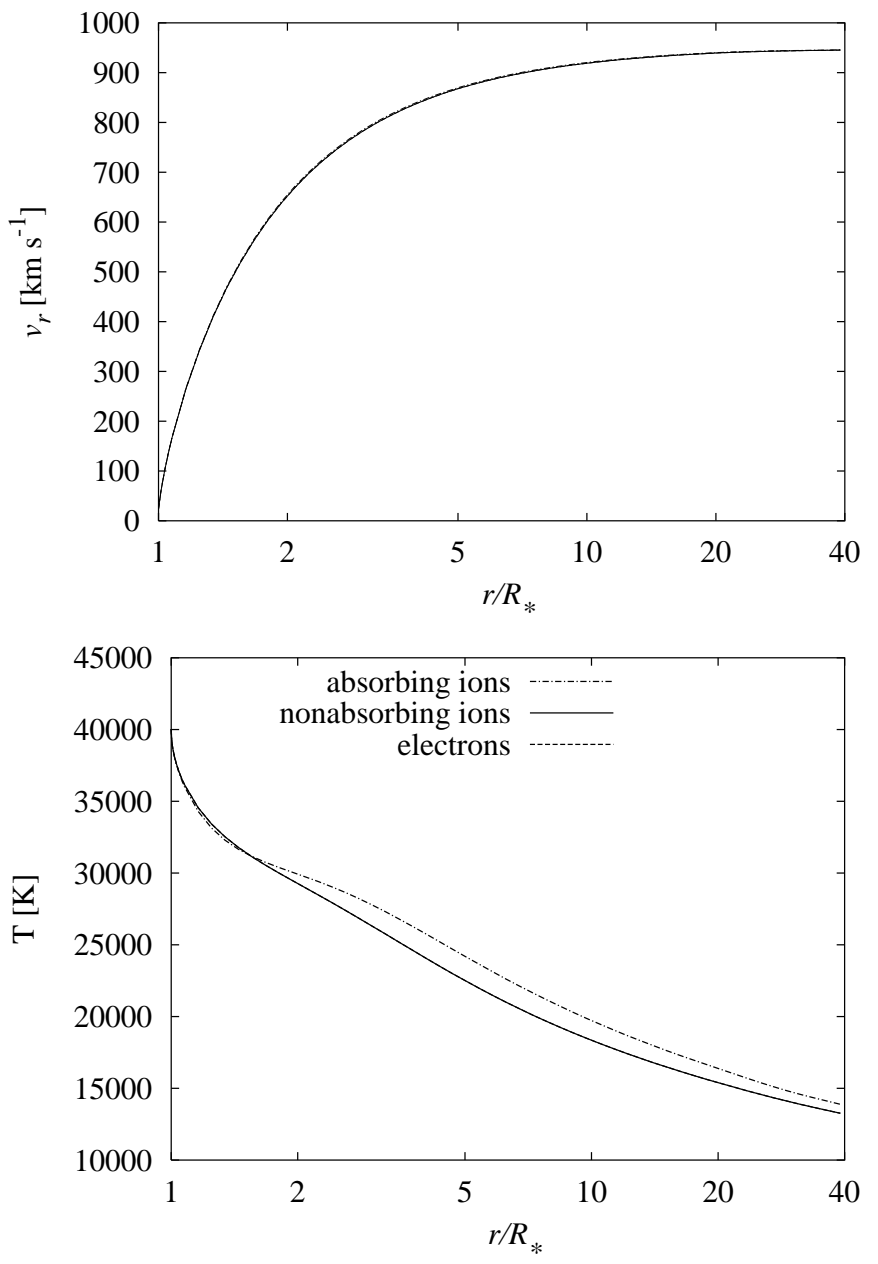

Fig. 2. The calculated model of a star with $\log L / L_{\odot}=6.57, T_{\text {eff }}=$ $50000 \mathrm{~K}$ and $Z / Z_{\odot}=0.001$. The stellar wind temperature structure is modified by frictional and GO heating. The temperature of absorbing ions is slightly higher than the temperature of other wind components. Note that velocities and densities of nonabsorbing component and electrons are nearly the same.

influence of these effects. Each of this model groups will be discussed separately.

\subsection{Modified temperature structure}

For stellar winds with only mild maximal velocity difference $\left(v_{r \mathrm{i}}-v_{r \mathrm{p}}\right) / \sqrt{\frac{2 k T}{m_{\mathrm{p}}}} \approx 0.1$ the only effect of the multicomponent flow is a slightly modified temperature structure compared to one-component models. Because the radiative force for models in this paper does not depend on the temperature, the outflow velocity is the same as for the one-component models. This is a difference compared to KKII where for this type of stars lowering of the outflow velocity occurs due to the lowering of the radiative acceleration.

An example of such models is given in Fig. 2. In this case the temperature structure is affected by the frictional and GO heating. However, the increase of the wind temperature is lower, compared to models of main-sequence stars with similar velocity difference, mainly due to the higher wind density and consequently larger radiative cooling. The wind velocity is not changed compared to the one-component case due to the neglected temperature dependence of the radiative force.

\subsection{Runaway instability in the outer region}

For stars with lower metallicity the wind density of absorbing component is lower and, thus, the velocity difference between wind components is higher in order to maintain common flow (see Eq. (6)). The lower the density, the higher the frictional heating. However, this picture changes for extremely low-density winds when the velocity difference is comparable to the sound speed, $\left(v_{r \mathrm{i}}-v_{r \mathrm{p}}\right) / \sqrt{\frac{2 k T}{m_{\mathrm{p}}}} \approx 1$. The wind is not stable in this case for the ionic Abbott waves any more (see Owocki \& Puls 2002; Krtička \& Kubát 2002) and the runaway instability occurs. This case did not occur in KKII because the temperature dependence of radiative force prevented the velocity difference to attain higher values comparable to the sound speed.

One of the models where such instability occurs is given in Fig. 3. Note that the outer model boundary is selected in such a way that the possible region of runaway instability is downstream.

\subsection{Possible fallback of nonabsorbing component}

For stars with even lower density, decoupling occurs in the region where the velocity of the nonabsorbing component is lower than the escape velocity. This causes either the fallback of hydrogen and helium onto the stellar surface or possible creation of some kind of clouds above the stellar surface (similar to that suggested by Porter \& Skouza 1999). Note that, in this case, time-dependent modeling is necessary to calculate the proper structure of a multicomponent stellar wind.

These conclusions are demonstrated in Fig. 4. The absorbing component dynamically decouples from the nonabsorbing component, accompanied even by decoupling of the temperatures.

\subsection{Formula for the maximal velocity difference}

To test the reliability of our approximate formula for the maximal velocity difference (6) we compared the approximate velocity differences with model ones for stars with negligible multicomponent effects. The results are given in Fig. 5. Clearly, formula (6) gives faithful predictions for the maximal drift velocity between absorbing and nonabsorbing wind components.

\section{Conclusions}

For most of the stellar wind models (moderate metallicity) calculated by Kudritzki (2002) we did not find any significant multicomponent effects, our calculations being in good agreement with his. Thus, radiatively driven winds of these stars can be adequately described by one-component models as done by Kudritzki. However, for some low metallicity stars we found that the multicomponent wind nature has a large effect and must be included to describe stellar winds of such stars. 

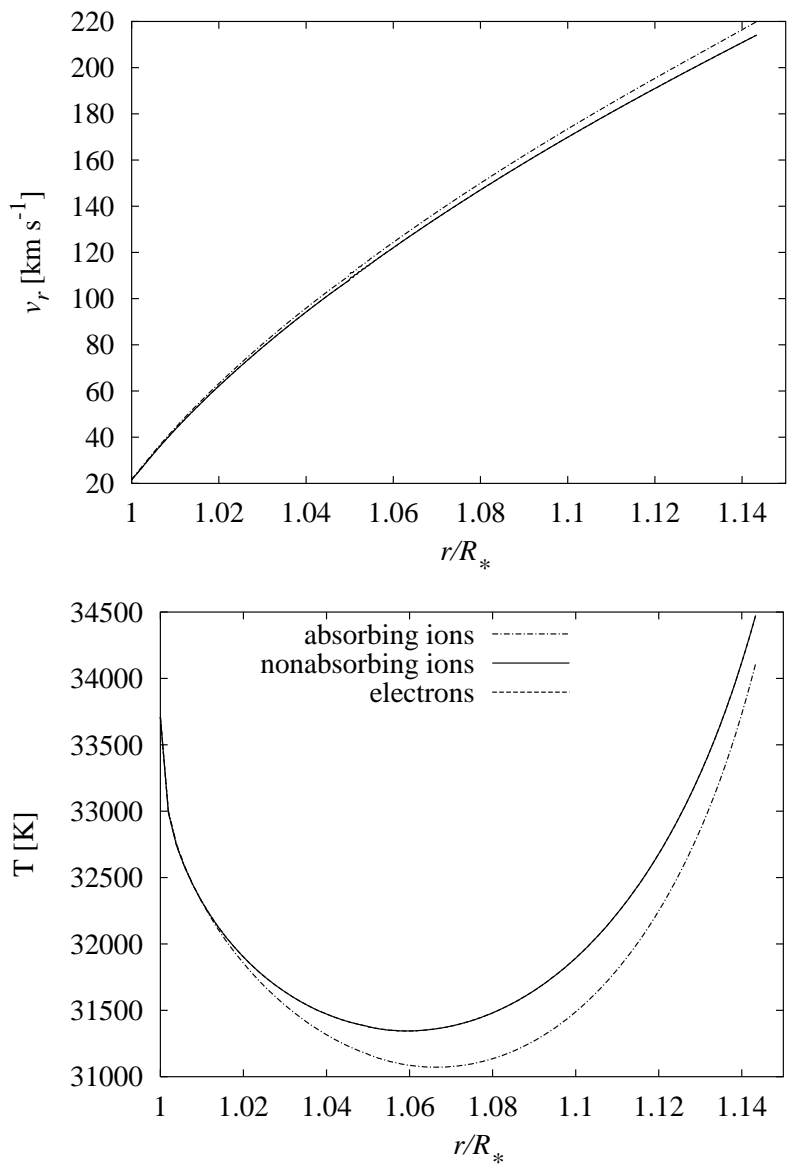

Fig. 3. Calculated model of a star with $\log L / L_{\odot}=6.42, T_{\text {eff }}=$ $40000 \mathrm{~K}$ and $Z / Z_{\odot}=0.001$. In the outer parts of the wind the velocity difference is comparable to the sound speed, $\left(v_{r \mathrm{i}}-v_{r \mathrm{p}}\right) / \sqrt{\frac{2 k T}{m_{\mathrm{p}}}} \approx 1$ and runaway instability occurs. Thus, the outer model boundary is below this area. The GO heating has a negative sign near the stellar surface and positive in the outer wind regions. Due to this variation the temperature of absorbing ions is lower than the temperature of other components.

The importance of multicomponent effects increases with decreasing metallicity and for any given star we are able to find a metallicity value below which the multicomponent effects significantly influence the wind structure. We found several consequences of multicomponent effects, ranging from modified temperature structure to runaway instability and possible fallback of the nonabsorbing component. Compared to results obtained by KKII, the frictional and GO heating are not so effective mainly due to the larger radiative cooling of higher density winds. Note that the changed wind structure influences the stellar radiative flux and may be specially important in the UV or X-ray region. Finally, due to their different terminal velocities these stars cannot be used for stellar distance measurements using the wind momentum-luminosity relation (cf. Kudritzki et al. 1999).

The validity of models presented here is influenced by some simplifications used. Although they do not influence the basic results, the real picture of multicomponent flows may be slightly different. Firstly, the radiative force is influenced by the temperature due to the temperature dependence of ionization
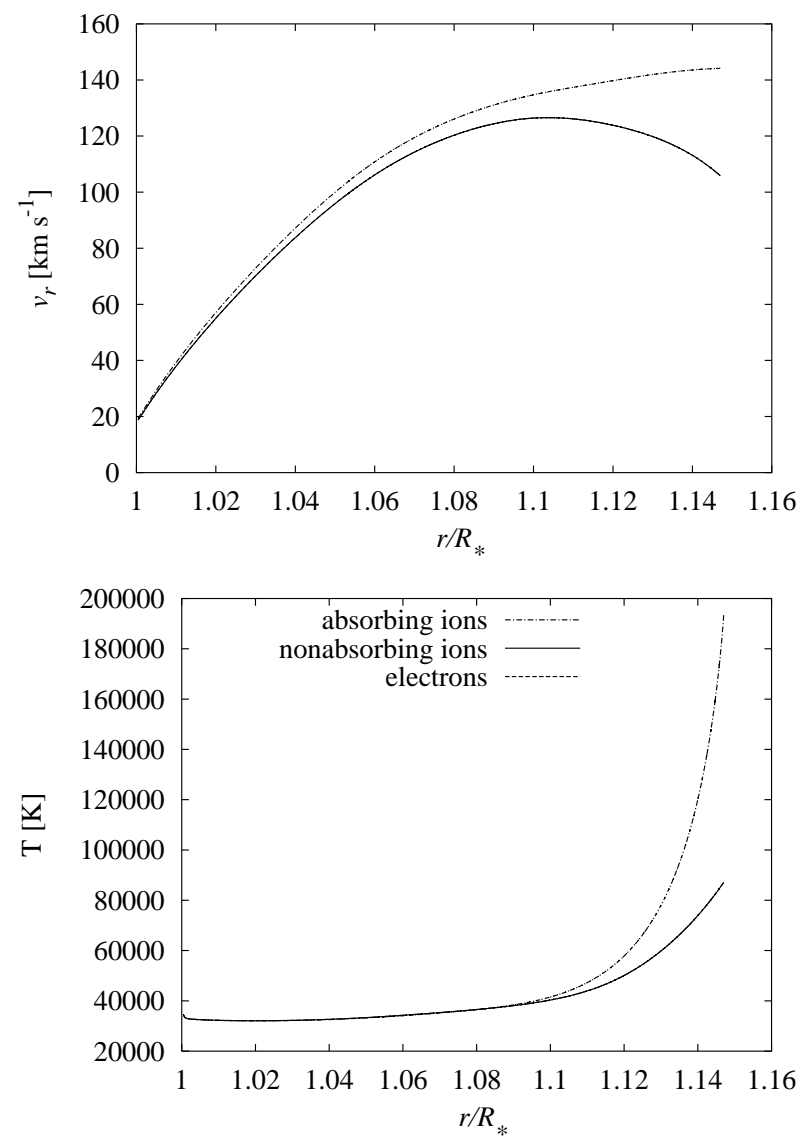

Fig. 4. Calculated model of a star with $\log L / L_{\odot}=6.30, T_{\text {eff }}=$ $40000 \mathrm{~K}$ and $Z / Z_{\odot}=0.001$. For this star the absorbing component is not able to accelerate the nonabsorbing component and the nonabsorbing component either form clouds around the star or falls back to the stellar surface. The increase of temperature is caused by the frictional and GO heating. Note that velocities and densities of nonabsorbing component and electrons are nearly the same.

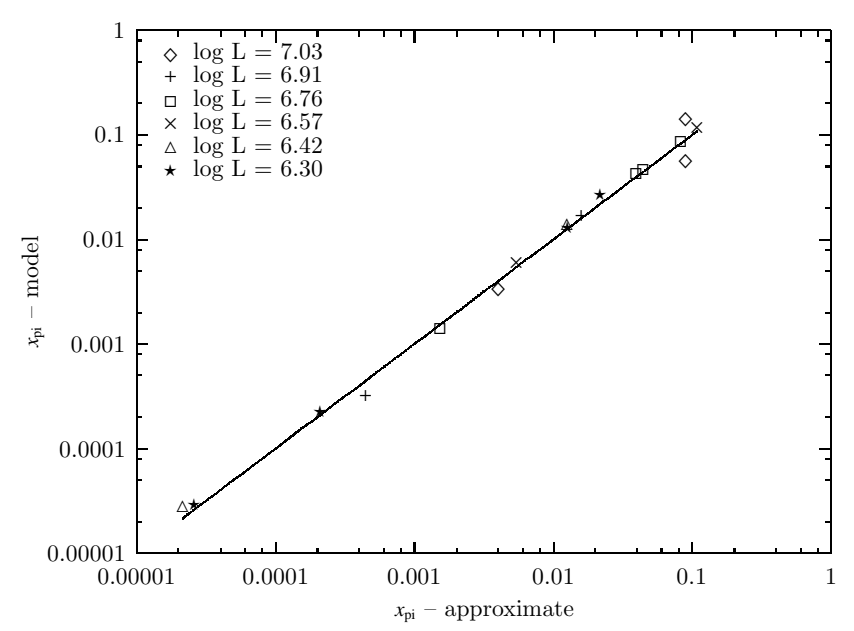

Fig. 5. Comparison of the maximal velocity difference calculated with approximate formula (6) and model ones.

and excitation. Although in the case of line-driven winds the occupation numbers are mainly given by the radiative processes, the temperature may be important especially in the 
case when large frictional heating occurs. For precise calculation of the radiative force it would be necessary to solve NLTE rate equations in the stellar wind. Secondly, the radiative heating/cooling term may be influenced by complex ionization and recombination processes. Finally, for extremely-low density stellar winds there may be an insufficient amount of metallic optically thick lines and $\mathrm{H}$, He lines may become important. We plan to adress these interesting issues using our NLTE wind code. First results obtained using this code for normal stars are promising and will be published elsewhere (Krtička \& Kubát, in preparation).

Acknowledgements. We are grateful to Dr. R. P. Kudritzki for discussion of his models. This research has made use of NASA's Astrophysics Data System. This work was supported by a PPARC Rolling Grant and by grants GA ČR 205/01/0656 and 205/02/0445, and by projects K2043105 and Z1003909.

\section{References}

Abbott, D. C. 1982, ApJ, 259, 282

Bromm, V., Coppi, P. S., \& Larson, R. B. 2002, ApJ, 564, 23

Burgers, J. M. 1969, Flow equations for composite gases (New York: Academic Press)
Castor, J. I., Abbott, D. C., \& Klein, R. I. 1975, ApJ, 195, 157 (CAK)

Castor, J. I., Abbott, D. C., \& Klein, R. I. 1976, in Physique des mouvements dans les atmosphères stellaires, ed. R. Cayrel, \& M. Sternberg (Paris: CNRS), 363

Friend, D. B., \& Abbott, D. C. 1986, ApJ, 311, 701

Gayley, K. G., \& Owocki, S. P. 1994, ApJ, 434, 684 (GO)

Gayley, K. G. 1995, ApJ, 454, 410

Krtička, J. 2002, in Stellar Atmosphere Modelling, ed. I. Hubeny, D. Mihalas, \& K. Werner, ASP Conf. Ser., submitted

Krtička, J., \& Kubát, J. 2001, A\&A, 377, 175 (KKII)

Krtička, J., \& Kubát, J. 2002, A\&A, 388, 531

Kubát, J., Puls, J., \& Pauldrach, A. W. A. 1999, A\&A, 341, 587

Kubát, J. 2002, in Modelling of Stellar Atmospheres, IAU Symp. 210, ed. N. E. Piskunov, W. W. Weiss, \& D. F. Gray, ASP Conf. Ser., submitted

Kudritzki, R. P. 2002, ApJ, 577, 389

Kudritzki, R. P., Puls, J., Lennon, D. J., et al. 1999, A\&A, 350, 970

Nakamura, F., \& Umemura, M. 2002, ApJ, 569, 549

Owocki, S. P., \& Puls, J. 2002, ApJ, 568, 965

Pauldrach, A., Puls, J., \& Kudritzki, R. P. 1986, A\&A, 164, 86

Porter, J. M., \& Skouza, B. A. 1999, A\&A, 344, 205

Puls, J., Kudritzki, R.-P., Herrero, A., et al. 1996, A\&A, 305, 171

Puls, J., Springmann, U., \& Lennon, M. 2000, A\&AS, 141, 23

Siess, L., Livio, M., \& Lattanzio, J. 2002, ApJ, 570, 329

Springmann, U. W. E., \& Pauldrach, A. W. A. 1992, A\&A, 262, 515 